\title{
METRIC SPACES WITH GEODESIC RICCI CURVES. I
}

\author{
JACK LEVINE
}

1. Introduction. The problem of determining all Riemannian spaces of three dimensions admitting geodesic Ricci curves has been solved by G. Ricci* and P. Walberer $†$ using, however, different methods. Although they obtained all such $V_{3}$, the complete explicit determination of all such $V_{n}$ for $n>3$ does not seem possible because of the increased number and complexity of the differential equations which arise.

In this paper the following two problems related to the above problem will be considered.

In the first problem we suppose given a set of linearly independent vectors $\ddagger \lambda_{a \mid}^{i}$ and wish to determine necessary and sufficient conditions on the $\lambda_{a \mid}^{i}$ in order that a set of scalars $\theta_{a}(\neq 0)$ exist which will define a metric space $V_{n}$ with a metric determined by

$$
g^{i j}=\sum_{h} e_{h} \bar{\lambda}_{h \mid}^{i} \bar{\lambda}_{h \mid}^{j}
$$

where

$$
\bar{\lambda}_{a \mid}^{i}=\theta_{a} \lambda_{a \mid}^{i}
$$

and $e_{h}(= \pm 1)$ are arbitrary; and such that the congruences of curves defined by the $\lambda_{a \mid}^{i}$ will be geodesics in the $V_{n}$ thus determined. (The vectors $\bar{\lambda}_{a \mid}^{t}$ define the same congruences as do the $\lambda_{a \mid}^{t}$, and these congruences form an orthogonal ennuple in the $V_{n}$.)

In the second problem we assume that these conditions on the $\lambda_{a \text { | }}^{i}$ have been determined and that the $n$ congruences defined by à set of $\lambda_{a \mid}^{i}$ are geodesics in the $V_{n}$ determined by

$$
g^{i j}=\sum_{h} e_{h} \lambda_{h \mid}^{i} \lambda_{h \mid}^{j}
$$

we then find necessary and sufficient conditions that, with respect to the metric (1), the congruences be geodesic Ricci curves.

* G. Ricci, Sulle varieta a tre dimensioni dotate die terne principali di congruenze geodetiche, Rendiconti della Reale Accademia dei Lincei, (5), vol. 27 (1918), pp. 21-28, 75-87.

$\dagger$ P. Walberer, Riemannsche Raume mit geodätischen Riccikurven, Hamburger Abhandlungen, vol. 10 (1934), pp. 152-168.

$\ddagger$ All indices take the values $1,2, \cdots, n$ unless otherwise noted. 
The method of Walberer is followed, and we obtain generalizations to $n$ dimensions of his conditions for 3 dimensions.*

2. Geodesic congruences. In this section we solve the first of the two problems stated in the introduction.

An orthogonal ennuple of unit vectors, $\lambda_{a \mid}^{i}$, of a $V_{n}$ will determine geodesic congruences if $\dagger$

$$
\gamma_{i a a}=0, \quad a \text { not summed, }
$$

where

$$
\gamma_{l h k} \equiv \lambda_{l, i, j} \lambda_{h \mid}^{i} \lambda_{k \mid}^{j}
$$

are the Ricci coefficients of rotation. $\ddagger$

The congruences are determined from the equations

$$
\frac{d x^{1}}{\lambda_{a \mid}^{1}}=\frac{d x^{2}}{\lambda_{a \mid}^{2}}=\cdots=\frac{d x^{n}}{\lambda_{a \mid}^{n}},
$$

and are defined by the $\lambda_{a \mid}^{i}$ to within a scalar factor, that is, the $\bar{\lambda}_{a \mid}^{i}$ given by (2) will define the same congruences as will the $\lambda_{a \mid}^{i}$. If then we wish to determine our conditions on the $\lambda_{a \mid}^{i}$ which make these congruences geodesics with respect to (1) we form equations (3) with $\lambda_{a \mid}^{i}$ replaced by $\bar{\lambda}_{a \mid}^{i}$ given by (2). The resulting equations in $\theta_{a}$ as unknowns must have solutions and this requirement leads to the desired conditions on the $\lambda_{a \mid}^{i}$.

We begin by replacing (3) by more suitable equations. If the operators $\Delta_{a}$ are defined by

$$
\Delta_{a} \equiv \lambda_{a \mid}^{i} \frac{\partial}{\partial x^{i}}
$$

their integrability conditions take the form $\S$

$$
\left(\Delta_{a}, \Delta_{b}\right) \equiv \Delta_{a} \Delta_{b}-\Delta_{b} \Delta_{a}=\sum_{i} e_{i}\left(\gamma_{i a b}-\gamma_{i b a}\right) \Delta_{i} .
$$

We write this in the form

$$
\left(\Delta_{a}, \Delta_{b}\right)=\stackrel{k}{c_{a b} \Delta_{k}}
$$

* New conditions are also obtained which do not appear for $n=3$.

$\dagger$ L. P. Eisenhart, Riemannian Geometry, p. 100. References to this book will be in the form RG.

‡ RG, pp. 97-98.

§ RG, p. 99. 
so that

$$
\stackrel{k}{c_{i j}}=e_{k}\left(\gamma_{k i j}-\gamma_{k j i}\right)=-c_{j i}^{k}
$$

These equations can be solved to give

$$
\gamma_{i j k}=\frac{1}{2}\left(e_{i} c_{j k}^{i}+e_{j} c_{k i}^{j}+e_{k} c_{j i}^{k}\right) .
$$

In terms of the $c$ 's the conditions (3) for geodesics become

$$
c_{i j}^{j}=0, \quad j \text { not summed. }
$$

We also remark, for later use, that the Jacobi identities

$$
\left(\Delta_{i},\left(\Delta_{j}, \Delta_{k}\right)\right)+\left(\Delta_{j},\left(\Delta_{k}, \Delta_{i}\right)\right)+\left(\Delta_{k},\left(\Delta_{i}, \Delta_{j}\right)\right)=0
$$

are equivalent to the conditions

$$
\Delta_{i} c_{j k}^{l}+\Delta_{j} c_{k i}^{l}+\Delta_{k} c_{i j}^{l}=-\left(c_{j k}^{h} c_{i h}^{l}+c_{k i}^{h} c_{j h}^{l}+c_{i j}^{h} c_{k h}^{l}\right) .
$$

Writing

$$
\bar{\Delta}_{a}=\theta_{a} \Delta_{a}
$$

we have

$$
\left(\bar{\Delta}_{a}, \bar{\Delta}_{b}\right)=\bar{c}_{a b}^{k} \bar{\Delta}_{k}
$$

from which we obtain

$$
\begin{aligned}
\bar{c}_{i j}^{k}=\frac{\theta_{i} \theta_{j}}{\theta_{k}} c_{i j}^{k}, & k \neq i, j, \\
\bar{c}_{i j}^{i}=\theta_{j}\left(c_{i j}^{i} \dot{-} \mu_{i j}\right), & i \neq j,
\end{aligned}
$$

where

$$
\mu_{i}=\log \theta_{i}, \quad \mu_{i j}=\Delta_{j} \mu_{i} .
$$

The conditions (6) for geodesics in the barred quantities are $\bar{c}_{i j}^{j}=0$, ( $j$ not summed), and from (9) these conditions become

$$
\mu_{i j}=\Delta_{j} \mu_{i}=c_{i j}^{i}, \quad i \neq j .
$$

These equations in the $\theta_{a}$ as unknowns must have solutions* and their integrability conditions give us our conditions on the $\lambda_{a \mid}^{i}$.

Forming the integrability conditions for (10), we obtain

* Of course, we do not assume $c_{i j}^{i}=0,(i$ not summed $)$, as this condition need only be satisfied by $\bar{c}_{i j}^{i}$. 


$$
\left(\Delta_{i}, \Delta_{j}\right) \mu_{k}=\Delta_{i} c_{k j}^{k}-\Delta_{j} c_{k i}^{k}=\stackrel{h}{c_{i j} \Delta_{h} \mu_{k}, \quad k \neq i, j, ~}
$$

or

$$
c_{i j}^{k} \Delta_{k} \mu_{k}=\Delta_{i} c_{k j}^{k}-\Delta_{j} c_{k i}^{k}-c_{i j}^{h} c_{k h}^{k}, \quad k \neq i, j ; k \text { not summed. }
$$

If in (7) we put $l=k$ (without summing), and transpose appropriate terms, we obtain

$$
\Delta_{i} c_{k j}^{k}-\Delta_{j} c_{k i}^{k}-c_{i j}^{h} c_{k h}^{k}=\Delta_{k} c_{i j}^{k}+c_{j k}^{h} c_{i h}^{k}+c_{k i}^{h} c_{j h}^{k}, \quad k \text { not summed. }
$$

Substitution from this equation into (11) gives

$$
\stackrel{k}{c_{i j}^{k} \Delta_{k} \mu_{k}}=\Delta_{k} c_{i j}^{k}+c_{j k}^{h} c_{i h}^{k}+c_{k i}^{h} c_{j h}^{k}, \quad k \neq i, j ; k \text { not summed. }
$$

The equations (12) are the desired integrability conditions of (10). If $c_{i j}^{k}=0,(k \neq i, j)$, for every $k$, equations (12) are satisfied identically. We thus assume that for each $k$ there is at least one $c_{i j}^{k} \neq 0$. We can then write (12) in the form*

$$
\Delta_{k} \mu_{k}=\frac{1}{c_{i j}^{k}}\left(\Delta_{k} c_{i j}^{k}+c_{j k}^{h} c_{i h}^{k}+c_{k i c}^{h} c_{j h}^{k}\right), \quad k \neq i, j,
$$

and equations (13) are to hold for all $i, j$ such that $c_{i j}^{k} \neq 0$.

Since the left member of (13) is independent of $i, j$, so must be the right member, that is, we must have

$$
\frac{1}{c_{i j}^{k}}\left(\Delta_{k} c_{i j}^{k}+c_{j k}^{h} c_{i h}^{k}+c_{k i c}^{h} c_{j h}^{k}\right)=\frac{1}{c_{a b}^{k}}\left(\Delta_{k} c_{a b}^{k}+c_{b k}^{h} c_{a h}^{k}+c_{k a}^{h} c_{b h}^{k}\right) \equiv H^{k}
$$

Equations (14) are to hold for all $i, j, a, b$ such that

$$
c_{i j}^{k} \neq 0, \quad c_{a b}^{k} \neq 0, \quad k \neq i, j, a, b .
$$

We now consider (13) and (10) combined, and form their integrability conditions. The only new conditions are obtained from $\left(\Delta_{l}, \Delta_{k}\right) \mu_{k}$, these conditions reducing to

$$
H^{k l} \equiv \Delta_{l} H^{k}-\Delta_{k} c_{k l}^{k}-c_{l k}^{h} c_{k h}^{k}+c_{k l}^{k} H^{k}=0, \quad k \neq l .
$$

If for given $k, i, j,(k \neq i, j), c_{i j}^{k}=0$, equations (12) reduce to

$$
H_{i j}^{k} \equiv c_{j k}^{h} c_{i h}^{k}+c_{k i}^{h} c_{j h}^{k}=0,
$$

which must be satisfied for all such $k, i, j$.

* If for any given $k, c_{i j}^{k}=0$, for all $i$ and $j$, the corresponding equation is identically zero and may be dropped. 
Under (8), (9), we find that the various $H$ 's transform in the following manner:

$$
\begin{aligned}
\bar{H}^{k} & =\theta_{k}\left(H^{k}-\mu_{k k}\right), & \bar{H}_{(i j)(a b)}^{k} & =\theta_{k} H_{(i j)(a b)}^{k}, \\
\bar{H}^{k l} & =\theta_{k} \theta_{l} H^{k l}, & \bar{H}_{i j}^{k} & =\theta_{i} \theta_{j} H_{i j}^{k} .
\end{aligned}
$$

The quantities $H_{(i j)(a b)}^{k}$ are the result of transposing the right member of (14) to the left, giving $H_{(i j)(a b)}^{k}=0$.

The quantities $H^{k l}$ are the generalizations to $n$ dimensions of the corresponding quantities for $n=3$ given by Walberer. The other $H$ 's do not appear for $n=3$.

We can state the following theorem:

Given a set of independent vectors $\lambda_{a \mid}^{i}$, necessary and sufficient conditions that a set of scalars $\theta_{a}$ should exist such that the vectors $\bar{\lambda}_{a \mid}^{i}=\theta_{a} \lambda_{a \mid}^{i}$ determine an orthogonal ennuple of geodesic congruences in the metric space defined by

$$
g^{i j}=\sum_{h} e_{h} \bar{\lambda}_{h \mid}^{i} \bar{\lambda}_{h \mid}^{j}
$$

are

$$
\begin{array}{rlr}
H^{k l} & =0, & k \neq l, \\
H_{(i j)(a b)}^{k}=0, & k \neq i, j, a, b, \\
H_{i j}^{k}=0, & k \neq i, j .
\end{array}
$$

Conditions (a) are used for all indices $k$ for which $H^{k}$ is defined. Conditions (b) are used only for indices $i, j, a, b,(\neq k)$, such that $c_{i j}^{k}=0$, $c_{a b}^{k}=0$. Conditions (c) are used only for indices $k, i, j$ such that $c_{i j}^{k}=0$, $(k \neq i, j)$.

It is to be noted that if $c_{i j}{ }^{i}=0,(i$ not summed), these integrability conditions are satisfied identically (as is to be expected). In this case $H^{k}=0$, as is seen from (7) on putting $k=l$. Then (a) and (b) follow, and (c) follows from the Jacobi identities (7).

From the definitions of the $H$ 's it is seen that the conditions (a), (b), (c) are independent of the $e_{h}$.

3. Geodesic Ricci curves. We consider now the second of the two problems stated in the introduction. We suppose given a set of $\lambda_{a \text { | }}^{i}$ satisfying conditions (a), (b), (c) of section 2 . Then there exists a set of the $\theta_{a}$ such that $\bar{c}_{i j}^{i}=0$, ( $i$ not summed), and thus we obtain the $\bar{\lambda}_{a \mid}^{i}$ of (2). We now drop the bars and suppose given a set of $\lambda$ 's such 
that $c_{i j}^{i}=0,(i$ not summed $)$, and wish to find a set of scalars $\theta_{a}(\neq 0)$ such that $\bar{\lambda}_{a \mid}^{i}=\theta_{a} \lambda_{a \mid}^{i}$ will determine the geodesic Ricci curves of a $V_{n}$ defined by (1).

An orthogonal ennuple of unit vectors, $\lambda_{a}^{i}$, of a $V_{n}$ will define the $n$ Ricci congruences of the $V_{n}$ if

$$
R_{i j} \lambda_{a \mid}^{i} \lambda_{b \mid}^{j}=0, \quad a \neq b,
$$

where $R_{i j}$ are the components of the Ricci tensor.*

From

$$
\begin{aligned}
\gamma_{l p q r} \equiv & R_{h i j k} \lambda_{l \mid}^{h} \lambda_{p \mid}^{i} \lambda_{q \mid}^{j} \lambda_{r \mid}^{k}=\Delta_{r} \gamma_{l p q}-\Delta_{q} \gamma_{l p r} \\
& +\sum_{m} e_{m}\left[\gamma_{l p m}\left(\gamma_{m q r}-\gamma_{m r q}\right)+\gamma_{m l r} \gamma_{m p q}-\gamma_{m l q} \gamma_{m p r}\right]
\end{aligned}
$$

and

$$
R_{i j}=g^{h k} R_{h i j k},
$$

we obtain as conditions equivalent to (18),

$$
\sum_{a} e_{a} \gamma_{a b c a}=0, \quad b \neq c .
$$

If we assume (3) satisfied (condition for geodesics), conditions (20) reduce to

$$
\sum_{a, m} e_{a}\left(\Delta_{a} \gamma_{a b c}+e_{m} \gamma_{a b m} \gamma_{m c a}\right)=0, \quad b \neq c .
$$

If in (7) we put $l=k$, and sum, and use (6), we obtain

$$
\Delta_{k} c_{i j}^{k}=0 .
$$

If now we substitute from (5) in (21) and make use of (6) and (22) we eventually reduce (21) to the form

$$
\begin{aligned}
& \sum_{a} e_{a}\left(e_{b} \Delta_{a} c_{a c}^{b}+e_{c} \Delta_{a} c_{a b}^{c}\right) \\
& \quad=\sum_{a, m} e_{a} e_{m}\left[e_{a} e_{m} c_{b m}^{a} c_{c a}^{m}+\stackrel{a}{a} c_{b m}^{a} c_{c m}^{a}-\frac{1}{2} e_{b} e_{c} c_{a m}^{b} c_{a m}^{c}\right], \quad b \neq c .
\end{aligned}
$$

We must impose conditions (23) and (6) in the $\bar{c}$ to obtain geodesic Ricci curves.

From (10) we now have

$$
\mu_{i j}=\Delta_{j} \mu_{i}=0, \quad i \neq j .
$$

* RG, pp. 110, 114. 
Hence,

$$
\left(\Delta_{i}, \Delta_{j}\right) \mu_{k}=\Delta_{i} \mu_{k j}-\Delta_{j} \mu_{k i}=0=c_{i j}^{m} \Delta_{m} \mu_{k}=c_{i j}^{k} \Delta_{k} \mu_{k}, \quad k \neq i, j,
$$

or

$$
\stackrel{k}{c_{i j} \mu_{k k}}=0, \quad k \neq i, j ; k \text { not summed. }
$$

The Jacobi conditions in the $\bar{c}$ add no new conditions on the $\theta_{a}$, for from (7) in the $\bar{c}$ we get (24) and (25) again.

We now form (23) in the $\bar{c}$ and thus get the remaining conditions on the $\theta$. We find on forming (23) in the $\bar{c}$ and then substituting from (8) and (9) that the resulting conditions can be written in the form

$$
\begin{aligned}
& \sum_{a} e_{a} \theta_{a}^{2}\left[\left(e_{b} \theta_{c}^{2} c_{a c}^{b}+e_{c} \theta_{b}^{2} c_{a b}^{c}\right) \mu_{a a}+e_{b} \theta_{c}^{2} \Delta_{a} c_{a c}^{b}+e_{c} \theta_{b}^{2} \Delta_{a} c_{a b}^{c}\right] \\
& =\sum_{a, m} e_{a} e_{m}\left[e_{a} e_{m} \theta_{b}^{2} \theta_{c}^{2} c_{b m}^{a} c_{c a}^{m}+\frac{\theta_{b}^{2} \theta_{c}^{2} \theta_{m}^{2}}{\theta_{a}^{2}} c_{b m}^{a} c_{c m}^{a}-\frac{1}{2} e_{b} e_{c} \theta_{a}^{2} \theta_{m}^{2} c_{a m}^{b} c_{a m}^{c}\right], \\
& b \neq c .
\end{aligned}
$$

The conditions on the $\theta_{i}$ are given by (24), (25), and (26). We shall solve the simplest case here, that is, the one in which all the $c$ 's are zero but one, which we may assume to be $c_{23}^{1}$. (If all the $c$ 's are zero, the space is flat.)

From (25) we see $\mu_{11}=0$, which together with (24) gives

$$
\theta_{1}=k_{1}=\text { const. }
$$

The only conditions obtained from (26) are

$$
\begin{aligned}
& \mu_{33}+\Delta_{3} A=0, \\
& \mu_{22}+\Delta_{2} A=0,
\end{aligned}
$$

where

$$
\stackrel{1}{c_{23}}=a, \quad A=\log a .
$$

Since now

$$
\left(\Delta_{i}, \Delta_{j}\right)=c_{i j}^{1} \Delta_{1}
$$

we have

$$
\left(\Delta_{i}, \Delta_{j}\right)=0, \quad i, j \neq 2 .
$$

Hence the operators $\Delta_{i},(i \neq 2)$, define an abelian group, ${ }^{*}$ and by a change of coordinate system, we have the canonical form

* L. P. Eisenhart, Continuous Groups of Transformations, p. 49. 


$$
\begin{aligned}
& \Delta_{i}=\frac{\partial}{\partial x^{i}}, \\
& \Delta_{2}=\alpha\left(x^{2}, x^{3}\right) \frac{\partial}{\partial x^{1}}+\frac{\partial}{\partial x^{2}},
\end{aligned}
$$$$
i \neq 2 \text {, }
$$

and

$$
a=-\frac{\partial \alpha}{\partial x^{3}}
$$

Equations (24), (29), (30) now show that

$$
\mu_{i}=\mu_{i}\left(x^{i}\right), \quad \text { or } \quad \theta_{i}=\theta_{i}\left(x^{i}\right),
$$

and then from (27) and (28) we obtain $\partial^{2} A / \partial x^{2} \partial x^{3}=0$. This gives

$a=B\left(x^{2}\right) C\left(x^{3}\right), \mu_{2}^{\prime}=-\frac{B^{\prime}}{B}, \mu_{3}^{\prime}=-\frac{C^{\prime}}{C}, \alpha=-B D, D=\int C d x^{3}$,

or

$$
\theta_{2}=\frac{k_{2}}{B}, \quad \theta_{3}=\frac{k_{3}}{C}
$$

Then $\bar{c}_{23}^{1}=k$ (=const.), and the $\bar{\Delta}_{a}$, by means of the coordinate transformation

$$
x^{\prime 1}=\frac{x^{1}}{k_{1}}, \quad x^{\prime 2}=\int \frac{B}{k_{2}} d x^{2}, \quad x^{\prime 3}=\frac{D}{k_{3}}, \quad x^{\prime i}=\int \frac{d x^{i}}{\theta^{i}}, \quad i>3,
$$

can be reduced to the canonical form (by dropping primes)

$$
\begin{array}{ll}
\bar{\Delta}_{i}=\frac{\partial}{\partial x^{i}}, & i \neq 2, \\
\bar{\Delta}_{2}=-k x^{3} \frac{\partial}{\partial x^{1}}+\frac{\partial}{\partial x^{2}} . &
\end{array}
$$

From (17) we have

$g^{i j}=e_{i} \delta_{j}^{i},(i, j \neq 1,2) ; g^{11}=e_{1}+e_{2}\left(k x^{3}\right)^{2}, g^{12}=-k e_{2} x^{3}, g^{22}=e_{2}$, from which the $g_{i j}$ are easily obtained.

North Carolina State Collegge 